\title{
Kinetics of solid state phase transformations: Measurement and modelling of some basic issues
}

\author{
S RAJU* and E MOHANDAS \\ Physical Metallurgy Division, Materials Development and Characterisation Group, \\ Indira Gandhi Centre for Atomic Research, Kalpakkam 603102 \\ e-mail: sraju@igcar.gov.in
}

\begin{abstract}
A brief review of the issues involved in modelling of the solid state transformation kinetics is presented. The fact that apart from the standard thermodynamic parameters, certain path variables like heating or cooling rate can also exert a crucial influence on the kinetic outcome is stressed. The kinetic specialties that are intrinsic to phase changes proceeding under varying thermal history are enumerated. A simple and general modelling methodology for understanding the kinetics of non-isothermal transformations is outlined.
\end{abstract}

Keywords. Phase transformation; kinetics; modelling.

\section{Introduction}

\subsection{Kinetic specialties of solid state phase changes}

A study of phase transformations that occur in solid state is of interest on both basic and applied grounds. Since phase transformations represent the explicit manifestation of thermodynamic instability, a comprehensive mapping of the evolutionary character of different phase fields in terms of appropriate intensive thermodynamic variables is extremely useful in constructing phase diagrams, the use of which in the design of materials is only too obvious. ${ }^{1}$ While thermodynamic considerations decide the ultimate stability of condensed systems, it is the time scale of the relaxation processes that marks the rate of attainment of this true thermodynamic equilibrium, which decides the effective usefulness of a solid as a practically useful engineering material. Testimonies to this fact abound in materials literature, especially in the realm of metals and alloys. While thermodynamics classifies phase changes in terms of the nature of discontinuity in appropriate susceptibility functions, the kinetic considerations serve to broaden this classification further. ${ }^{2}$ The number of kinetic paths available in general for solid state phase transformations is many and these can be realised by selecting and tuning appropriate kinetic path variable. ${ }^{3}$ Figure 1 schematically illustrates this point

*For correspondence with respect to one kinetic variable, namely heating or cooling rate $(\beta)$. The possible other kinetic path variables include: starting grain size $(d)$ or morphology of the material, stress state $(\sigma)$, irradiation level, etc. These could act either singly or in combination to decide the overall kinetics of transformation. In the present article, an attempt is made to highlight the role of one important kinetic variable, namely the heating or cooling rate in decisively altering the kinetics of simple structural transformations that occur in metals and alloys. The choice of this variable is based on the pre-eminent status that thermal

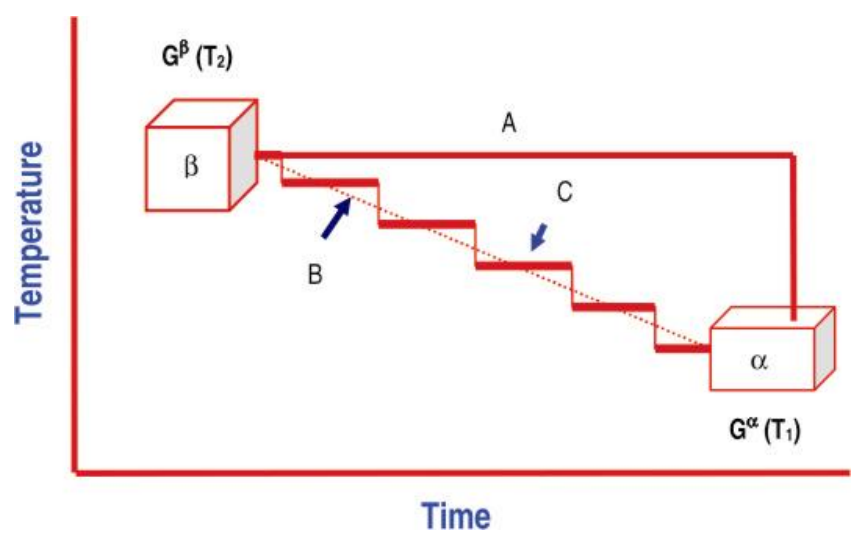

Figure 1. A schematic of different kinetic path ways offered by three different cooling histories is shown. Paths $A$ and $B$ stand for transformations occurring under isothermal and linear cooling conditions, where as the path $\mathrm{C}$ represents a step-wise cooling with small incremental isothermal holds. Note that in all the three paths A, B and $\mathrm{C}$ the net reduction in Gibbs energy $\Delta^{\circ} \mathrm{G}$ is the same. 
history as a process parameter enjoys in dictating the final microstructure of many engineering alloys used in strategic applications. The other reason is that relevant experimental kinetic data with sufficient degree of accuracy are easily generated by taking recourse to high resolution thermal analysis techniques, the results of which are rather easily processed to obtain information on transformation temperatures, the transformation velocity, especially their dependence on thermal history. In what follows, we highlight some interesting, but somewhat less well-known kinetic aspects of simple structural transformations through select examples drawn from our recent research.

\section{Isothermal versus non isothermal processes: Influence of heating and cooling rates}

\subsection{Isothermal phase change}

As illustrated in figure 1, a phase transformation between two phases $\alpha$ and $\beta$ can be realised under varying time $(t)$-temperature $(T)$ combinations, known under the generic name of thermal history. In the purely isothermal case, the sample is held at a fixed temperature (path A in figure 1) the magnitude of which decides effectively the available chemical driving force $(\Delta G)$ and diffusional mobility $(D)$ of atoms. Apart from temperature, the time duration of isothermal holds decides the extent of transformation. For the case of $\alpha \rightarrow \beta$ transformation which is promoted by an increase of temperature, both $\Delta G$ and the diffusional mobility $D$ of atoms increase with the extent of overheating, $T-T_{\text {tr }} T_{\text {tr }}$ is the equilibrium transformation temperature at which $\Delta G$ goes identically to zero. The temperature dependent Gibbs energy difference to a first order approximation may be given as, ${ }^{4}$

$$
\Delta^{\circ} G(T) \approx \Delta^{\circ} H_{\mathrm{tr}} \Delta T / T_{\mathrm{tr}},
$$

$\Delta^{\circ} H_{\text {tr }}$ is the latent heat associated with the phase change at $T_{\mathrm{tr}}$, and $\Delta T=\left|T-T_{\mathrm{tr}}\right|$ is the degree of overheating (or undercooling, if $\beta \rightarrow \alpha$ reverse transformation is considered). The fraction transformed is recorded as a function of time $X(t)$, for time spans that are greater than a minimum threshold known as the incubation time, $\tau_{\text {inc }}$ (figure 2 ). It is clear that for $t<\tau_{\text {inc }}, X(t)=0 . \tau_{\text {inc }}$ is a characteristic kinetic quantity the magnitude of which is decided by both $\Delta G(T)$ and diffusivity. ${ }^{5}$
The basic isothermal experimental data are $\{X, t\} \tau$ and $(\partial X / \partial t)_{\mathrm{T}}$, both of which are obtainable by a combination of thermal analysis and metallographic techniques. Besides the standard transformation plots (figure 2), a most popular way of representing $\{X, t\}_{\mathrm{T}}$ data is by means of isotransformation fraction $X\{T, t\}$ contour plots, which are often called TTT (time, temperature, transformation) diagrams. One such diagram is schematically sketched in figure 3 for the $\beta \rightarrow \alpha$ transformation. It is useful to note

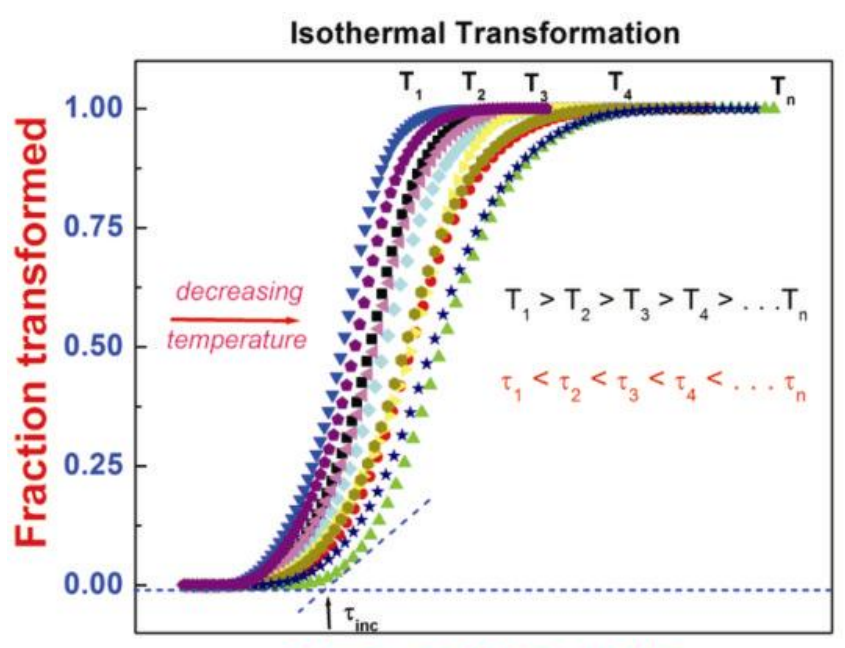

Time, arbitrary units

Figure 2. A graphical portrayal of the isothermal transformation diagram is shown. Note the $S$-type nature of the transformation profiles and also the dependence of incubation time on reaction temperature.

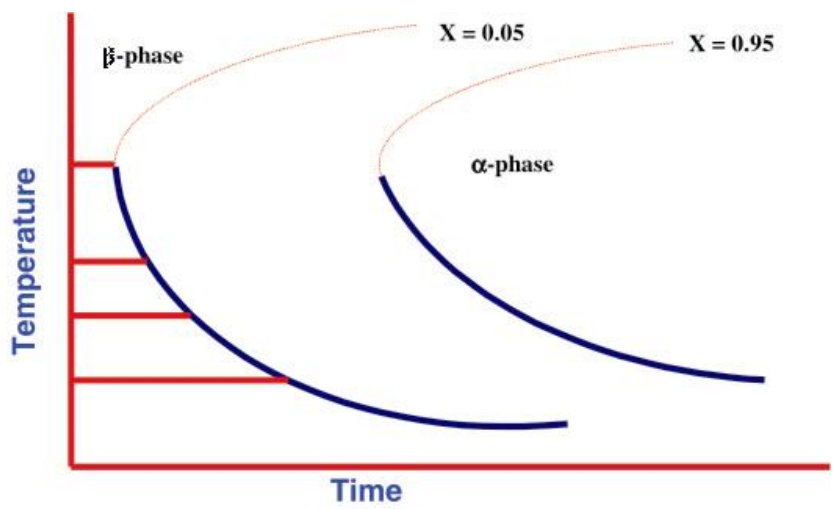

Figure 3. A schematic of TTT diagram for the formation of the low temperature stable $\alpha$ phase from the high temperature polymorph, $\beta$ is shown. The horizontal lines parallel to the time axis represent the time to reach fixed extent of transformation. Note the $C$-type nature of this isotransformation contours. The upper portion of this contour, indicated in red is however absent for the reverse $\alpha \rightarrow \beta$ phase change. Refer to text for further details. 
that the $C$-type nature of the isotransformation contour is intrinsic to phase changes occurring during cooling and is generally absent for the corresponding $\alpha \rightarrow \beta$ on-heating phase change. The reason is that although $\Delta G$ increases with undercooling $\left|T-T_{\mathrm{tr}}\right|$, this is accompanied by reduced atomic mobility which in turn retards the transformation. ${ }^{3}$

\subsection{Non-isothermal phase change}

The isochronal process involves continuous heating or cooling as a function of time (path B in figure 1) or with interrupted isothermal holds (path $\mathrm{C}$ in figure 1). To avoid unnecessary complexity, we shall consider in the present analysis only linear heating and cooling processes. A typical continuous heating transformation plot is portrayed in figure 4 . It is clear that higher heating rates push the transformation onset temperatures $\left(T_{\mathrm{S}}\right)$ to successively higher values; stated in equivalent terms, for a fixed temperature $T$, the transformation is more advanced for slower heating rates. A corollary of this deduction is that the adoption of successively higher heating rates takes the transforming system increasingly away from equilibrium. The case of equilibrium in this case pertains to the asymptotic limit, $\beta \rightarrow 0$. Thus, although the system is physically taken to successively higher temperatures in quick succession in isochronal experiments, it is done so without realising the equilibrium extent of transformation

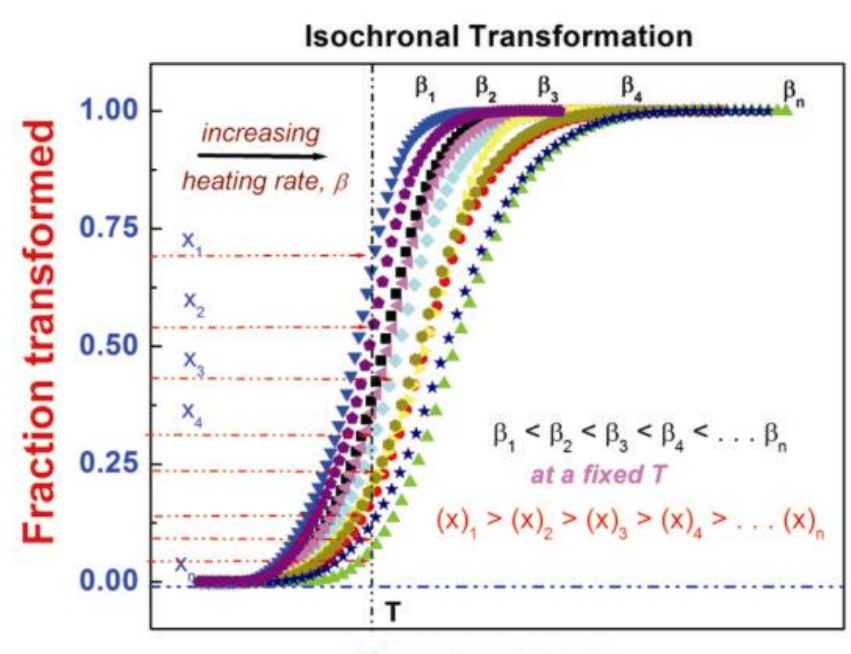

Temperature

Figure 4. The isochronal or continuous heating transformation plot is graphically illustrated. Note that increasing the heating rate pushes up the transformation start temperature. Also, for fixed temperature, the transformation is more advanced at lower heating rates. slated for that temperature. This lagging behind the equilibrium means a pileup of unspent driving force $\Delta G(T)$ which is only waiting to be dissipated through alternate modes of phase transformation. There is one evidences in metallurgical literature, which suggest that on rapid heating or cooling conditions, classical nucleation and growth mediated phase changes are inhibited and yield way to martensitic or displacive types. ${ }^{6,7}$

From the experimental point of view, the heating or cooling rate dependence of transformation temperatures, in addition to the transformed fraction $X(T)$, offer a valuable wholesome kinetic data set. It is generally the case that the transformation temperatures are highly nonlinear with respect to heating or cooling rate, and this tendency is exhibited more remarkably by the transformation finish $\left(T_{\mathrm{f}}\right)$ temperatures. ${ }^{8}$ An example to this effect is provided in figure 5 , which incidentally represents a continuous heating transformation (CHT) diagram for the $\alpha \rightarrow \beta$ structural change in uranium. ${ }^{9}$ It is interesting to note that the temperature interval of the two phase domain $\alpha+\beta$ increases at higher heating rates, a feature which is unique to kinetic phase diagrams.

\section{Modelling of non-isothermal transformation kinetics: Additivity approximation}

It is generally the case that non-isothermal experiments are easily performed with minimal expendi-

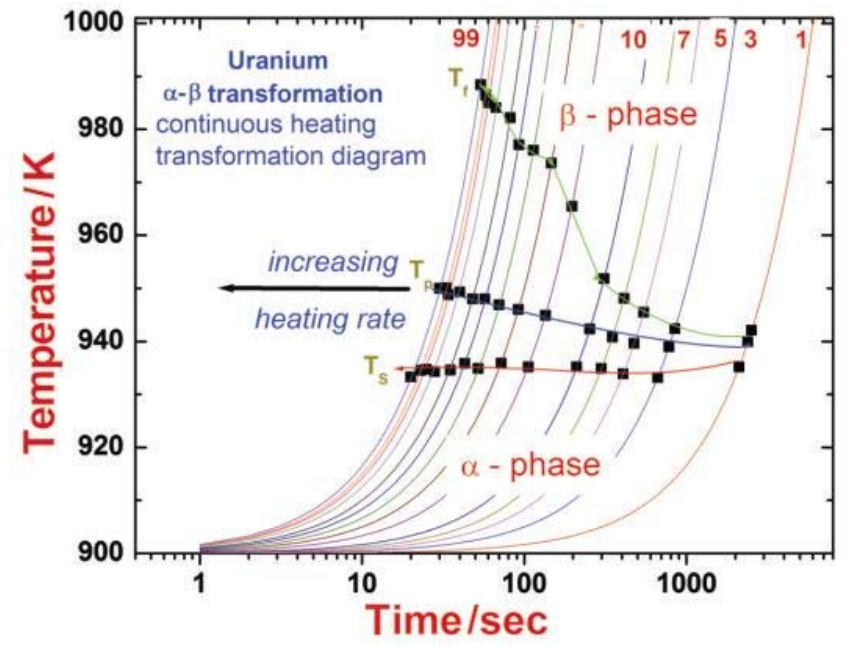

Figure 5. The continuous heating transformation (CHT) diagram for the $\alpha \rightarrow \beta$ transformation in uranium is shown. The dotted lines represent various heating rates, on to which the measured transformation start $\left(T_{\mathrm{S}}\right)$, peak $\left(T_{\mathrm{P}}\right)$ and finish $\left(T_{\mathrm{f}}\right)$ temperatures are marked. Note the increased width of the $\alpha+\beta$ two phase domain at higher heating rates. 
ture of time; but their results are the difficult ones to understand and interpret in concrete terms. The isothermal experiments, while more involved in terms of labour, offer fundamental results that may be tested in terms of transparent theoretical descriptions. ${ }^{10-13}$ From an applied context, most of the industrial heat treatments are done under complex thermal history, thereby necessitating a holistic understanding of both isothermal and non-isothermal phase transformation kinetics. In literature, there are very few rigorous treatments aimed primarily at tackling the problem of phase evolution under varying time-temperature history ${ }^{14-16}$ and generally their mathematical complexity comes in the way of their widespread practical appeal.

Traditionally, the problem of non-isothermal transformation kinetics is modelled as a sum of short isothermal steps; this approximation is popularly called the Scheil's additivity rule in metallurgy ${ }^{17}$, which even as of now is extensively used in modelling the solidification kinetics of alloys, homogenisation as well as particle dissolution upon heating and precipitate formation reactions under cooling, etc. ${ }^{18-20}$ The essence of the additivity approximation is illustrated in path $\mathrm{C}$ in figure 1. Basically, the total time span, $t=\left(T_{\mathrm{f}}-T_{\mathrm{S}}\right) / \beta$, spent between the two temperature intervals $T_{\mathrm{f}}$ and $T_{\mathrm{s}}$ under a fixed scan rate $\beta$, is divided into $n$ number of small isothermal steps with time interval $\Delta t$. Mathematically, the total fraction transformed $X(T)$ at any particular temperature $T\left(T_{\mathrm{S}} \leq T \leq T_{\mathrm{f}}\right)$ which is reached after a finite time interval $t=T-T_{\mathrm{S}} / \beta$, is approximated as

$$
X(T)=X\left(T_{\mathrm{S}}+\beta t\right)=\sum x_{i} ; \quad(i=1, n) .
$$

Further

$$
t=\sum \Delta t_{\mathrm{i}}, \quad \text { for } i=1, n .
$$

The individual $x_{i}\left(\Delta t_{i}\right)$, the isothermally transformed fractions are determined normally by adopting a well-behaved isothermal rate equation to model the progress of transformation. There are no firm guidelines as to the correct choice of the isothermal rate equation, but the one proposed by Kolmogorov, Johnson, Mehl and Avrami for diffusional phase changes ${ }^{21}$ has been extensively used. This isothermal KJMA model is given as below.

$$
X(t)=1-\exp \left\{-\left((k(T) t)^{n}\right\} .\right.
$$

$k(T)$ represents the temperature dependent isothermal rate constant, which as a matter of convenience is taken to be of the Arrhenius form.

$$
k(T)=k_{0} \exp \left(-Q_{\text {eff }} / R T\right)
$$

$n$ is the transformation exponent. $k_{0}$ and $Q_{\text {eff }}$ assume standard implications. In principle, the rate constant $k$ and the transformation exponent $n$ are sensitive to transformation path variable, such as heating or cooling rate to varying degrees. This fact is often overlooked in simplistic modelling approaches however. ${ }^{22}$ But the major issue of modelling non-isothermal transformation kinetics does not lie in evolving a proper non-isothermal analogue of $\mathrm{KJMA}^{23}$ since more serious technical limitations arise from the use of the additivity principle itself. As this is an important issue, we shall dwell upon this in a little more detail here.

In invoking the linear superposition of isothermal events, it is implicitly assumed that the jump from one isothermal hold to the next one is instantaneous, and hence no phase formation is allowed or expected during this jump. It is nevertheless obvious that instantaneous jump is practically meaningless. The error accrued by this assumption may be minimised by going in for smaller $\Delta t_{i}$. The other assumption that is implicit in the additivity approximation is that different isothermal events are isokinetic, a preposition which is generally not obeyed by many solid state phase changes, especially during the later growth stages. ${ }^{24}$ The core of non-isokineticity stems from the fact that the starting configuration given to each individual isothermal transformation step is just not the same, as for example, in a single shot isothermal phase change. Since transformation kinetics are a strong function of the starting configuration, each successive isothermal step has somewhat altered kinetic imperatives and hence as a rule, solid state phase changes under non-isothermal conditions are non-isokinetic in nature.

\section{A simple non-additive model for non-isothermal phase change}

A practical implication of the above mentioned arguments is that the adoption of rate expressions that are originally developed for isothermal phase changes, for modelling of isochronal transformation kinetics is technically incorrect, notwithstanding the successful numerical fitting of the experimental data by such procedures. The classical isothermal rate equations are invariably the variable separable type, in the sense that $(\mathrm{d} X / \mathrm{d} t)$ is expressed as a product of two functions, one each for characterising the $T$ and $x$ dependence. 
$(\mathrm{d} X / \mathrm{d} t)=f(X) k(T)$

$f(X)$ is an empirical reaction model and $k(T)$ is the rate constant as mentioned before. But such a separable representation is conceptually incompatible with the basic premise of isochronal transformation kinetics, ${ }^{16}$ despite the fact that they are extensively patronised in data analysis, basically because of their simplicity.

On the other hand, a conceptually transparent alternative would be to start from the basic fact that for small range of values of $\beta\left(10^{0}\right.$ to $\left.10^{2} \mathrm{~K} \mathrm{~min}^{-1}\right)$ usually encountered in controlled thermal analysis experiments, the transformation start temperature is found to vary in a linear manner with $\beta$. That is,

$$
T_{\mathrm{S}}(\beta)=T_{\mathrm{o}}+\beta \tau \text {. }
$$

$T_{o}$ is the ideal thermodynamic equilibrium transformation temperature corresponding to the static case, $\beta \rightarrow 0$. In practice, $T_{\mathrm{o}}$ is treated as a fit parameter. $\tau$ is a characteristic time of the non-isothermal phase change kinetics, which is somewhat akin to the incubation time, $\tau_{\text {inc }}$ proposed for the isothermal case. The physical basis of (7) is rather straight forward. Given with adequate time $t$ greater than $\tau_{\text {inc }}$, the reaction would certainly start at $T_{o}$; but in a continuous heating experiment, the mandatory expenditure of a minimum of $\tau_{\text {inc }}$ seconds after having reached $T_{o}$ would alter the temperature to a higher value, namely $T_{o}+\beta \tau$. So the first traces of the transformation product are observed not at $T_{o}$, but only at $T_{o}+\beta \tau$. Certainly this is a conservative approximation. The actual time delay involved in realising advanced transformation stages is even more than that given by this linear model. This deficiency can be partly accounted for by now making $\tau$ itself a function of $\beta$. Thus by using a second tier of approximation,

$$
\tau(\beta)=\tau_{0}+\tau^{\prime} \beta
$$

the nonlinear variation of $T_{\mathrm{S}}$ with $\beta$ is readily obtained. The experimental validity of this model is amply borne out by the measured $\alpha \Leftrightarrow \beta$ transformation temperature of uranium as a function of $\beta{ }^{9}$ Figure 6 graphically portrays this point for various allotropic phase changes in titanium.

In the next phase, we shall propose a general analytical rate expression for $(\partial X / \partial t)_{\beta}$ that is valid for both isothermal and non-isothermal conditions. This is given as:

$$
(\partial X / \partial t)_{\beta}=X_{\mathrm{eq}}(T)-X(t) / \tau
$$

Equation (9) is based on the basic deduction that the instantaneous rate of transformation $(\partial X / \partial t)_{\beta}$ for a chosen $\beta$, is dependent on the extent of deviation of the transformation extent $X(t)$ from its corresponding equilibrium value at $T, X_{\mathrm{eq}}(T)$. This latter quantity that may be obtained from equilibrium phase diagram (lever rule) or may be set as an internal reference value which is obtained under very slow heating or cooling conditions. A rough estimate of $\tau$ (assumed to be $\beta$ independent in the trial estimation) can be obtained from the experimental data using (7). Thus $(\partial X / \partial t)_{\beta}$ is readily obtained. But what is more appealing is that (9) can be used in an inverse manner to obtain $X_{\mathrm{eq}}(T)$, the equilibrium fraction transformed from experimental data measured under dynamic conditions. But the actual caveat in realising this possibility lies in measuring accurately the $X(t)$ values; this method is expected to work better for larger values of $X_{\mathrm{eq}}(T)-X(t)$, which means that experiments need be performed under considerably large heating or cooling rates.

The essential theoretical import of (9) is that under non-isothermal conditions, $X(t)$ approaches $X_{\text {eq }}$ with a time constant given by $\tau$. For the sake of maintaining analogy with the isothermal case, we term this $\tau_{\beta}$ as a dynamic incubation time. It can be logically argued that $\tau_{\beta}$ exceeds $\tau$-static by a margin which is

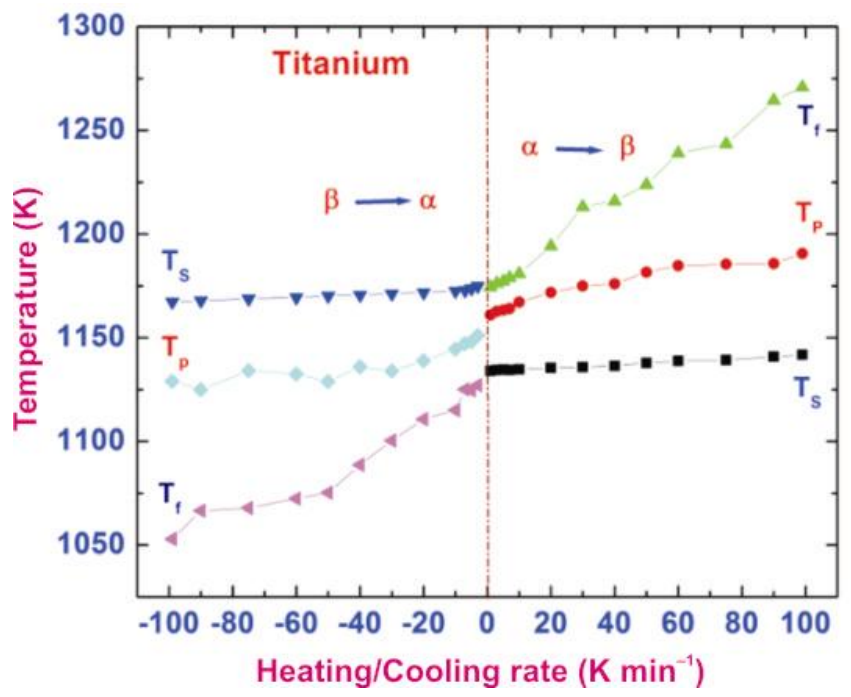

Figure 6. The influence of heating and cooling rates on the $\alpha \Leftrightarrow \beta$ structural transformations in titanium is graphically depicted. Note that compared to $T_{\mathrm{S}}$, the $T_{\mathrm{f}}$ temperature exhibits a higher degree of nonlinearity in its variation with $\beta$. 
actually the time delay introduced because of kinetic considerations. An accurate knowledge of the difference, $\tau_{\beta}-\tau$-static is very useful in interrelating the isothermal and non-isothermal transformation data, as for example argued by Rios. ${ }^{25}$ At present, the generalised functional dependence of $\tau_{\beta}$ with $\beta$ is not known. Nevertheless, an attempt is made to model the $\tau(\beta)$ behaviour through an exponential relaxation model.

$$
\tau=\tau_{0}+A \exp (-m / \beta)
$$

In the above description, $\tau$ approaches $\tau_{0}$, when $\beta \rightarrow 0 . m$ is an empirical parameter having the same dimension as that of $\beta$ and is characterising the exponential decay of $\tau$ with $1 / \beta$. Further, $\tau=\tau_{0}+A$ for $\beta \rightarrow \infty$. Thus, a limiting time is found to operate for very high heating rates. All these assume that $\tau$ versus $\beta$ is a continuous function; any abrupt change in the behaviour is suggestive of the operation of a different transformation mechanism. At present the theoretical justification for this linear + exponential model is not clear, but it is motivated by a similar experimental finding made with respect to the heating rate dependence of the rate constant $k$ for the structural transformation in uranium. ${ }^{9}$ Recently, we found that this model is found to fit the experimental data well for the $\beta \rightarrow \alpha$ transformation in pure titanium as well. ${ }^{26}$

For the sake of relating the exponential decay model of $\tau$ with a kinetic coefficient namely the effective activation energy $Q_{\text {eff }}$ of the transformation process, an empirical modification of (11) in the following manner is attempted.

$$
\tau=\tau_{0}+A \exp \left(-m^{\prime} Q_{\text {eff }} / \beta R T_{\mathrm{P}}\right)
$$

Note, that $Q_{\text {eff }} / R T_{\mathrm{P}}$ is a dimensionless quantity. Here, $T_{\mathrm{P}}$ is the peak transformation temperature which can be conveniently obtained by differential thermal analysis. Both (10) and (11) work well for the polymorphic structural changes in uranium and titanium. ${ }^{9,26}$. In figure 7 , the exponential relaxation model (10) of $\tau$ is illustrated using results obtained on $\beta \rightarrow \alpha$ structural transformation in a dilute titanium alloy.

As a passing remark, we may also state that (11) serves to pseudo linearise the $\beta$-dependence of experimental transformation temperature data. By taking logarithm of (11) and after some rearrangement of terms, we get

$$
\ln \left(\tau-\tau_{0}\right)=\ln A-m^{\prime} Q_{\text {eff }} / \beta R T_{P} .
$$

It is interesting to note that (12) bears interesting similarity with the Ozawa, Kissinger like linearization schemes used originally in the estimation of apparent activation energy values from thermal analysis data. ${ }^{27,28}$ The utility of (12) in obtaining a linear correlation is again illustrated in figure 8 for the case of titanium. The separation of $m^{\prime} Q_{\text {eff }}$ in to independent parameters is a non-trivial task; it is clear that both $m$ and $m^{\prime}$ are related. It has been

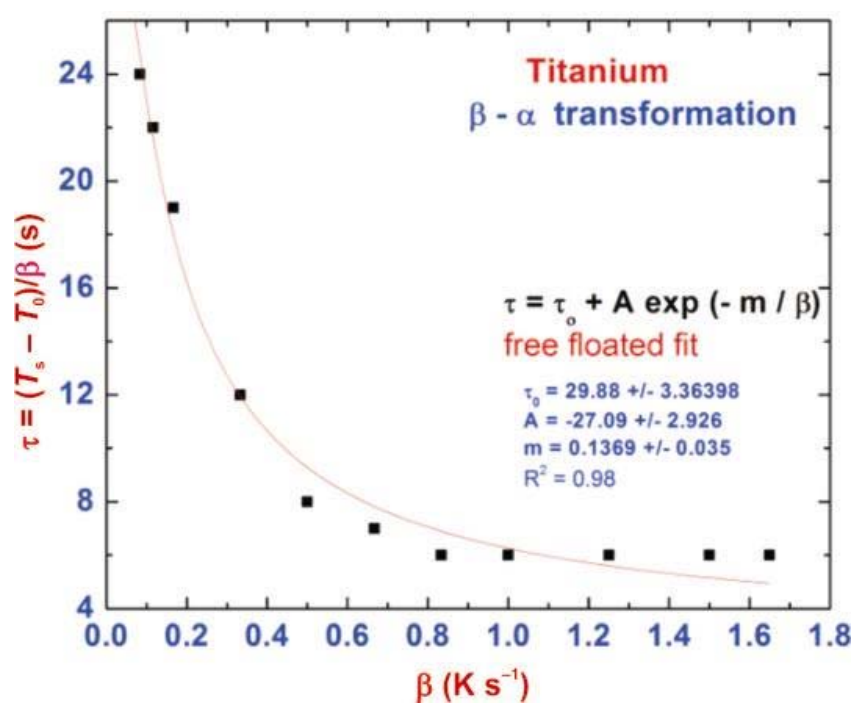

Figure 7. The exponential decay of the characteristic transformation time $\tau$ with cooling rate $\beta$, for the $\beta \rightarrow \alpha$ phase transformation in titanium is illustrated.

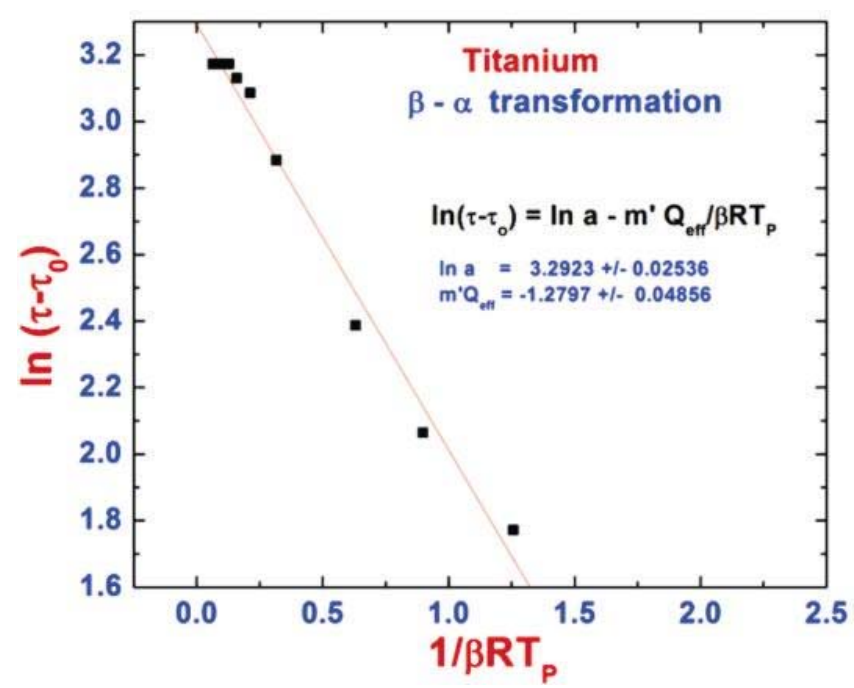

Figure 8. The linear scaling of the quantity $\ln \left(\tau-\tau_{0}\right)$ with $1 / \beta R T_{\mathrm{p}}$ is shown for the $\beta \rightarrow \alpha$ phase transformation in titanium. 
found in case of uranium that $m^{\prime} \approx m / 10$. Using this scaling, we estimate for titanium a value of about $100 \mathrm{~kJ} \mathrm{~mol}^{-1}$ for the $Q_{\text {eff }}$ of $\beta \rightarrow \alpha$ transformation.

As a final remark, we must also say that the present scheme is equally applicable to diffusional and displacive transformation types. This model is intrinsically capable of mirroring the kinetic asymmetry associated with forward and reverse transformations. The incorporation of other path variables like grain size is yet to be worked out. Besides, the role of internal and external stresses in modifying $\tau$ the characteristic time scale is also to be understood. These developments, when incorporated correctly can make this scheme a fully physically based one.

\section{Conclusions}

In the present paper, we have outlined certain topical issues that are of significance in understanding the kinetic imperatives of non-isothermal phase changes. In particular, the non-trivial role of thermal history as a kinetic path variable in triggering alternate transformation modes is highlighted. This is followed by the limitations of the additivity approximation that is widely adopted in switching over from isothermal to non-isothermal reference frames. Finally, a simple model based on experimental findings of non-isothermal transformations is proposed that removes most of obscurities associated with additivity approximation.

\section{Acknowledgements}

The authors acknowledge Mr B Jeya Ganesh, Mr Arun Kumar Rai and Mrs Josephine Prabha for their invaluable experimental support during various stages of this work. The sustained encouragement received from Drs Vijayalakshmi, K B S Rao, P R Vasudeva Rao and Baldev Raj is sincerely acknowledged.

\section{References}

1. Rhines F 1951 Phase diagrams in metallurgy, their development and applications (NY: McGraw Hill)
2. Hillert M 1998 Phase equilibrium, phase diagrams and phase transformations: their thermodynamic basis (Cambridge: Cambridge University Press)

3. Jena A K and Chaturvedi M C 1992 Phase transformations in materials (New Jersey: Prentice-Hall) p 414

4. Turnbull D 1956 In Solid state physics: Advances in research and applications (eds) Seitz F and Turnbull D (NY: Academic Press) vol. 3, p 226

5. Porter D A and Easterling K E 1981 Phase transformations in metals and alloys (Workingham: Van Nostrand Reinhold)

6. Wilson E A 1984 Met. Sci. 18471

7. Rechtien J J and Nelson R D 1973 Metall. Trans. A4 2755

8. Burke J and Dixon P H 1962 J. Nucl. Mater. 738

9. Arun Kumar Rai, Raju S, Jeya Ganesh B, Mohandas E, Sudha S and Ganesan V 2009 J. Nucl. Mater. 383 215

10. Starink M J 2003 Thermochimica Acta 404163

11. Liu F, Sommer F, Bos C and Mittemeijer E J 2007 Int. Mater. Rev. 52193

12. Ya Lyubov B 1978 Kinetic theory of phase transformations (New Delhi: Amerind Publ. Co.)

13. Christian J W 1975 The theory of transformations in metals and alloys (Oxford: Pergamon Press) 2nd edn

14. Reti T, Gergely M and Tardy P 1987 Mater. Sci. Technol. 3365

15. Lusk M T and Jou H-J 1997 Metall. Trans. A28 287

16. Leblond J B and Mottet G, Devaux J and Devaux J-C 1985 Mater. Sci. Tech. 1815

17. Scheil E 1935 Archiv. fur das Eisenhuttenwesen 8 565; as cited in Ref. 25

18. Kruger P 1993 J. Phys. Chem. Solids 541549

19. Ye J S, Chang H B and Hsu T Y 2003 Metall. Trans. A34 1259

20. Kamat R G, Hawbolt E B, Brown L C and Brimacombe J K 1992 Metall. Trans. A23 2469

21. Cumbrera F L and Sanchez Bajo F 1995 Thermochimica Acta 266315

22. Meisel L V and Coate P J 1983 Acta Metall. 31 1053

23. Vasquez J, Wagner C, Villarez $\mathrm{P}$ and Jieminez-Garay R 1996 Acta Mater. 444807

24. Todinov M T 2000 Acta Mater. 484217

25. Rios P R 2005 Acta Mater. 534893

26. Prabha J 2009 unpublished research

27. Ruitenberg G, Woldt E and Petford-Long A K 2001 Thermochimica Acta $\mathbf{3 7 8} 97$

28. Baram J and Erukhimovitch V 1997 Thermochimica Acta 29181 